\title{
A New Three-Term Conjugate Gradient-Based Projection Method for Solving Large-Scale Nonlinear Monotone Equations
}

\section{Mompati Koorapetse and Professor Kaelo}

\section{University of Botswana}

Department of Mathematics, Private Bag UB00704, Gaborone

E-mail(corresp.): kaelop@mopipi.ub.bw

E-mail: sphetto77@gmail.com

Received January 22, 2019; revised September 1, 2019; accepted September 2, 2019

\begin{abstract}
A new three-term conjugate gradient-based projection method is presented in this paper for solving large-scale nonlinear monotone equations. This method is derivative-free and it is suitable for solving large-scale nonlinear monotone equations due to its lower storage requirements. The method satisfies the sufficient descent condition $F_{k}^{T} d_{k} \leq-\tau\left\|F_{k}\right\|^{2}$, where $\tau>0$ is a constant, and its global convergence is also established. Numerical results show that the method is efficient and promising.
\end{abstract}

Keywords: nonlinear monotone equations, derivative-free, global convergence.

AMS Subject Classification: 90C06; 90C30; 90C56; 65K05; 65K10.

\section{Introduction}

Conjugate gradient-based projection methods are a class of methods suited for solving large-scale nonlinear equations

$$
F(x)=0,
$$

where $F: \mathbb{R}^{n} \rightarrow \mathbb{R}^{n}$ is a continuous and monotone function. A function $F$ is said to be monotone if it satisfies

$$
(F(x)-F(y))^{T}(x-y) \geq 0, \quad \forall x, y \in \mathbb{R}^{n} .
$$

Nonlinear monotone equations arise in many practical applications, for example, as chemical equilibrium systems [13], economic equilibrium problems [5],

Copyright (c) 2019 The Author(s). Published by VGTU Press

This is an Open Access article distributed under the terms of the Creative Commons Attribution License (http://creativecommons.org/licenses/by/4.0/), which permits unrestricted use, distribution, and reproduction in any medium, provided the original author and source are credited. 
and signal and image recovery problems [8]. Some mathematical problems can also be transformed into finding the solution of problem (1.1), such as variational inequality problems. This wide application has seen a number of researchers study iterative schemes (methods) for solving monotone equations over the years.

Conjugate gradient methods $[3,9,17,22]$ are very efficient methods for solving large-scale unconstrained optimization problems mainly due to their simplicity and low storage requirements. This has over the years influenced researchers to propose conjugate gradient-based projection methods by combining conjugate gradient methods with the hyperplane projection method [16] to solve nonlinear monotone equations.

Conjugate gradient-based projection methods are iterative methods that generate the next iterate $x_{k+1}$, given $x_{k}$, by

$$
x_{k+1}=x_{k}-\frac{F\left(z_{k}\right)^{T}\left(x_{k}-z_{k}\right)}{\left\|F\left(z_{k}\right)\right\|^{2}} F\left(z_{k}\right),
$$

where $z_{k}=x_{k}+\alpha_{k} d_{k},\|\cdot\|$ denotes the Euclidean norm and

$$
d_{k}= \begin{cases}-F_{k}, & \text { if } k=0, \\ -F_{k}+\beta_{k} d_{k-1}, & \text { if } k \geq 1,\end{cases}
$$

with $\beta_{k}$ a parameter such that

$$
F_{k}^{T} d_{k} \leq-\tau\left\|F_{k}\right\|^{2}, \tau>0,
$$

$F_{k}=F\left(x_{k}\right)$ and $\alpha_{k}$ is a step length. One such method is that by Papp and Rapajić [14] who proposed a derivative-free projection method

$$
d_{k}= \begin{cases}-F_{k}, & \text { if } k=0, \\ -F_{k}+\beta_{k}^{F R} w_{k-1}-\theta_{k} F_{k}, & \text { if } k \geq 1,\end{cases}
$$

where

$$
\beta_{k}^{F R}=\frac{\left\|F_{k}\right\|^{2}}{\left\|F_{k-1}\right\|^{2}}, \quad \theta_{k}=\frac{\left\|F_{k}\right\|^{2}\left\|w_{k-1}\right\|^{2}}{\left\|F_{k-1}\right\|^{4}} \quad \text { and } \quad w_{k-1}=z_{k}-x_{k}=\alpha_{k} d_{k} .
$$

The global convergence of this method was established using the line search

$$
-F\left(z_{k}\right)^{T} d_{k} \geq \sigma \alpha_{k}\left\|F\left(z_{k}\right)\right\|\left\|d_{k}\right\|^{2},
$$

with $\sigma>0$ being a constant.

Another method is that by Sun and Liu [18] which proposes the search direction

$$
d_{k}= \begin{cases}-F_{k}, & \text { if } k=0 \\ -\left(1+\beta_{k} \frac{F_{k}^{T} d_{k-1}}{\left\|F_{k}\right\|^{2}}\right) F_{k}+\beta_{k} d_{k-1}, & \text { if } k \geq 1,\end{cases}
$$

where $\beta_{k}=t\left\|F_{k}\right\| /\left\|d_{k-1}\right\|$, for some positive constant $t$. They proved that the method is globally convergent and showed through numerical results that the method is very efficient. 
More recently, Liu and Feng [10] proposed a derivative-free iterative method

$$
d_{k}= \begin{cases}-F_{k}, & \text { if } k=0 \\ -\theta_{k} F_{k}+\beta_{k} d_{k-1}, & \text { if } k \geq 1\end{cases}
$$

where $\beta_{k}=\left\|F_{k}\right\|^{2} /\left(d_{k-1}^{T} w_{k-1}\right)$, with $w_{k-1}=y_{k-1}+t d_{k-1}, y_{k-1}=F_{k}-F_{k-1}$, $t=1+\max \left\{0,-\frac{d_{k-1}^{T} y_{k-1}}{\left\|d_{k-1}\right\|^{2}}\right\}$ and $\theta_{k}=\tau-\frac{F_{k}^{T} d_{k-1}}{d_{k-1}^{T} w_{k-1}}$, for some positive constant $\tau$ that satisfies (1.3). This method was applied on convex constrained monotone equations. For more conjugate gradient-based projection methods, the reader is referred to $[1,2,4,7,11,12,15,19,20,21]$.

In this paper, motivated by the work of Zheng and Zheng [22], we propose another conjugate gradient-based projection method. This method is presented in the next section. In Section 3, we prove the global convergence of the proposed method. Numerical results follow in Section 4 and conclusion is presented in Section 5.

\section{Algorithm}

Recently, in solving an unconstrained optimization problem

$$
\min _{x \in \mathbb{R}^{n}} f(x)
$$

where $f: \mathbb{R}^{n} \rightarrow \mathbb{R}$ is a continuously differentiable function, Zheng and Zheng [22] proposed two conjugate gradient methods that generate $d_{k}$ by

$$
d_{k}= \begin{cases}-g_{k}, & \text { if } k=0 \\ -g_{k}+\beta_{k} d_{k-1}, & \text { if } k \geq 1\end{cases}
$$

with $g_{k}=\nabla f\left(x_{k}\right)$ being the gradient of $f$ at $x_{k}$ and the parameter $\beta_{k}$ given by

$$
\begin{aligned}
\beta_{k}^{D H S D L} & =\frac{\left\|g_{k}\right\|^{2}-\frac{\left\|g_{k}\right\|}{\left\|g_{k-1}\right\|}\left|g_{k}^{T} g_{k-1}\right|}{\mu\left|g_{k}^{T} d_{k-1}\right|+d_{k-1}^{T} y_{k-1}}-t \frac{g_{k}^{T} s_{k-1}}{d_{k-1}^{T} y_{k-1}}, \\
\beta_{k}^{D L S D L} & =\frac{\left\|g_{k}\right\|^{2}-\frac{\left\|g_{k}\right\|}{\left\|g_{k-1}\right\|}\left|g_{k}^{T} g_{k-1}\right|}{\mu\left|g_{k}^{T} d_{k-1}\right|-d_{k-1}^{T} g_{k-1}}-t \frac{g_{k}^{T} s_{k-1}}{d_{k-1}^{T} y_{k-1}},
\end{aligned}
$$

where $y_{k-1}=g_{k}-g_{k-1}, \mu>1$ and $t>0$. This method was shown to converge globally using the strong Wolfe line search and it was also shown to perform very well numerically.

Now, motivated the definition of $\beta_{k}$ by Zheng and Zheng [22], we propose a new conjugate gradient-based projection method for (1.1) by defining the search direction as

$$
d_{k}= \begin{cases}-F_{k}, & \text { if } k=0 \\ -F_{k}+\beta_{k} d_{k-1}-\theta_{k} w_{k-1}, & \text { if } k \geq 1\end{cases}
$$


where

$$
\beta_{k}=\frac{\left\|F_{k}\right\|^{2}-\frac{\left\|F_{k}\right\|}{\left\|F_{k-1}\right\|}\left|F_{k}^{T} F_{k-1}\right|}{\mu\left\|F_{k}\right\|\left\|d_{k-1}\right\|-F_{k-1}^{T} d_{k-1}}, \quad \theta_{k}=\frac{F_{k}^{T} w_{k-1}}{\mu\left\|w_{k-1}\right\|^{2}}
$$

$w_{k-1}=y_{k-1}+d_{k-1}, y_{k-1}=F_{k}-F_{k-1}+r s_{k-1}, s_{k-1}=x_{k}-x_{k-1}, \mu>1$ and $r>0$. Note here that $w_{k-1}$ is defined as in $[10,11]$ except that in our case $t$ is always taken to be $t=1$. The specific steps of our proposed method are presented below in Algorithm 1.

Algorithm 1 [Three-term Conjugate Gradient-based Method (TCGM)].

1. Give $x_{0} \in \mathbb{R}^{n}$, the parameters $\sigma, \kappa, r, \mu$ and $\rho \in(0,1)$. Set $k=0$.

2. FOR $k=0,1, \ldots$ do

3. If $\left\|F_{k}\right\|=0$, then stop. Otherwise, go to Step 4 .

4. Compute $d_{k}$ by (2.1)-(2.2).

5. Compute $z_{k}=x_{k}+\alpha_{k} d_{k}$ where $\alpha_{k}=\max \left\{\kappa \rho^{i}: i=0,1,2, \ldots\right\}$ such that the inequality

$$
-F\left(x_{k}+\alpha_{k} d_{k}\right)^{T} d_{k} \geq \sigma \alpha_{k}\left\|d_{k}\right\|^{2}
$$

with $\sigma>0$ and $\kappa>0$ being constants, is satisfied.

6. If $\left\|F\left(z_{k}\right)\right\|=0$, then stop. Otherwise, compute $x_{k+1}$ using (1.2).

7. Set $k=k+1$ and go to Step 3 .

\section{ENDFOR}

Throughout this paper, we assume that the following assumption holds.

\section{Assumption 1.}

(i) The function $F(\cdot)$ is monotone on $\mathbb{R}^{n}$.

(ii) The function $F(\cdot)$ is Lipschitz continuous on $\mathbb{R}^{n}$, i.e. there exists a positive constant $L$ such that

$$
\|F(x)-F(y)\| \leq L\|x-y\|, \quad \forall x, y \in \mathbb{R}^{n} .
$$

(iii) The solution set of (1.1) is nonempty.

\section{Convergence analysis}

We now present the global convergence of our algorithm under Assumption 1.

Lemma 1. Suppose that Assumption 1 holds. Let the sequence $\left\{x_{k}\right\}$ be generated by Algorithm 1. Then the search direction $d_{k}$ satisfies the sufficient descent condition

$$
F_{k}^{T} d_{k} \leq-\left(1-\frac{1}{\mu}\right)\left\|F_{k}\right\|^{2}, \quad \forall k \geq 0 \quad \text { and } \quad \mu>1 .
$$


Proof. Since $d_{0}=-F_{0}$, we have $F_{0}^{T} d_{0}=-\left\|F_{0}\right\|^{2}$. From this, we get that $F_{0}^{T} d_{0} \leq-\left(1-\frac{1}{\mu}\right)\left\|F_{0}\right\|^{2}$ is satisfied for any $\mu>1$. To prove (3.1) for $k \geq 1$, we assume $F_{k-1}^{T} d_{k-1} \leq-\left(1-\frac{1}{\mu}\right)\left\|F_{k-1}\right\|^{2}$ and show it is true for $k$. Notice from the definition of $\beta_{k}$ that we have

$$
0 \leq \beta_{k} \leq \frac{\left\|F_{k}\right\|^{2}}{\mu\left\|F_{k}\right\|\left\|d_{k-1}\right\|} .
$$

Now, we obtain from (2.1)-(2.2) that

$$
\begin{aligned}
& F_{k}^{T} d_{k}=-\left\|F_{k}\right\|^{2}+\beta_{k} F_{k}^{T} d_{k-1}-\theta_{k} F_{k}^{T} w_{k-1} \\
& \quad=-\left\|F_{k}\right\|^{2}+\frac{\left\|F_{k}\right\|^{2}-\frac{\left\|F_{k}\right\|}{\left\|F_{k-1}\right\|}\left|F_{k}^{T} F_{k-1}\right|}{\mu\left\|F_{k}\right\|\left\|d_{k-1}\right\|-F_{k-1}^{T} d_{k-1}} F_{k}^{T} d_{k-1}-\frac{\left(F_{k}^{T} w_{k-1}\right)^{2}}{\mu\left\|w_{k-1}\right\|^{2}} \\
& \quad \leq-\left\|F_{k}\right\|^{2}+\frac{\left\|F_{k}\right\|^{2}}{\mu\left\|F_{k}\right\|\left\|d_{k-1}\right\|}\left\|F_{k}\right\|\left\|d_{k-1}\right\|=-\left(1-\frac{1}{\mu}\right)\left\|F_{k}\right\|^{2} .
\end{aligned}
$$

Hence (3.1) is satisfied for all $k \geq 0$.

The following lemma indicates that if the sequence $\left\{x_{k}\right\}$ is generated by Algorithm 1 and $x^{*}$ is such that $F\left(x^{*}\right)=0$, then the sequence $\left\{x_{k}-x^{*}\right\}$ is decreasing and convergent, thus the sequence $\left\{x_{k}\right\}$ is bounded.

Lemma 2. Suppose Assumption 1 holds and the sequence $\left\{x_{k}\right\}$ is generated by Algorithm 1. For any $x^{*}$ such that $F\left(x^{*}\right)=0$, we have that

$$
\left\|x_{k+1}-x^{*}\right\|^{2} \leq\left\|x_{k}-x^{*}\right\|^{2}-\left\|x_{k+1}-x_{k}\right\|^{2}
$$

and the sequence $\left\{x_{k}\right\}$ is bounded. Furthermore, either $\left\{x_{k}\right\}$ is finite and the last iterate is a solution of (1.1) or $\left\{x_{k}\right\}$ is infinite and

$$
\sum_{k=0}^{\infty}\left\|x_{k+1}-x_{k}\right\|^{2}<\infty
$$

which means

$$
\lim _{k \rightarrow \infty}\left\|x_{k+1}-x_{k}\right\|=0 .
$$

Proof. The conclusion follows from Theorem 2.1 in [16].

Since $F(x)$ is continuous and $\left\{x_{k}\right\}$ is a bounded sequence it follows that there exists a constant $M>0$ such that $\left\|F\left(x_{k}\right)\right\| \leq M, \forall k \geq 0$. Thus, $\left\{F_{k}\right\}$ is bounded.

Lemma 3. For all $k \geq 0$, we have

$$
\left(1-\frac{1}{\mu}\right)\left\|F_{k}\right\| \leq\left\|d_{k}\right\| \leq\left(1+\frac{2}{\mu}\right)\left\|F_{k}\right\| .
$$


Proof. From (3.1) and Cauchy-Schwarz inequality, we have

$$
\left\|d_{k}\right\| \geq\left(1-\frac{1}{\mu}\right)\left\|F_{k}\right\| .
$$

From (2.1)-(2.2) we have

$$
\begin{aligned}
\left\|d_{k}\right\| & \leq\left\|F_{k}\right\|+\left|\beta_{k}\right|\left\|d_{k-1}\right\|+\left|\theta_{k}\right|\left\|w_{k-1}\right\| \\
& \leq\left\|F_{k}\right\|+\frac{\left\|F_{k}\right\|^{2}}{\mu\left\|d_{k-1}\right\|\left\|F_{k}\right\|}\left\|d_{k-1}\right\|+\frac{\left\|F_{k}\right\|\left\|w_{k-1}\right\|}{\mu\left\|w_{k-1}\right\|^{2}}\left\|w_{k-1}\right\| \\
& =\left\|F_{k}\right\|+\frac{2}{\mu}\left\|F_{k}\right\|=\left(1+\frac{2}{\mu}\right)\left\|F_{k}\right\| .
\end{aligned}
$$

Therefore,

$$
\left(1-\frac{1}{\mu}\right)\left\|F_{k}\right\| \leq\left\|d_{k}\right\| \leq\left(1+\frac{2}{\mu}\right)\left\|F_{k}\right\| .
$$

Lemma 4. Let Assumption 1 hold and the sequences $\left\{x_{k}\right\}$ and $\left\{z_{k}\right\}$ be generated by Algorithm 1. Then, we have

$$
\alpha_{k} \geq \min \left\{\kappa, \frac{\rho \mu(\mu-1)}{(L+\sigma)(\mu+2)^{2}}\right\} \text {. }
$$

Proof. If $\alpha_{k} \neq \kappa$, then $\alpha_{k}{ }^{\prime}=\frac{\alpha_{k}}{\rho}$ does not satisfy (2.3), that is

$$
-F\left(x_{k}+\alpha_{k}{ }^{\prime} d_{k}\right)^{T} d_{k}<\sigma \alpha_{k}{ }^{\prime}\left\|d_{k}\right\|^{2} .
$$

This together with (2.3) and (3.1) imply that

$$
\begin{aligned}
(1- & \left.\frac{1}{\mu}\right)\left\|F_{k}\right\|^{2} \leq-F_{k}^{T} d_{k} \\
& =\left(F\left(x_{k}+\alpha_{k}^{\prime} d_{k}\right)-F_{k}\right)^{T} d_{k}-F\left(x_{k}+\alpha_{k}^{\prime} d_{k}\right)^{T} d_{k} \\
& \leq L \alpha_{k}^{\prime}\left\|d_{k}\right\|^{2}+\sigma \alpha_{k}^{\prime}\left\|d_{k}\right\|^{2}=(L+\sigma) \alpha_{k} \rho^{-1}\left\|d_{k}\right\|^{2} \\
& \leq(L+\sigma) \alpha_{k} \rho^{-1}\left(\frac{\mu+2}{\mu}\right)^{2}\left\|F_{k}\right\|^{2} .
\end{aligned}
$$

Thus $\alpha_{k} \geq \rho \mu(\mu-1) /(L+\sigma)(\mu+2)^{2}$.

Theorem 1. Suppose that Assumption 1 holds, and the sequence $\left\{x_{k}\right\}$ is generated by Algorithm 1. Then we have

$$
\lim _{k \rightarrow \infty} \inf \left\|F_{k}\right\|=0 .
$$

Proof. We assume that (3.3) does not hold, that is, $\exists \eta>0$ such that $\left\|F_{k}\right\| \geq$ $\eta, \forall k \geq 0$. From (3.1) and Cauchy-Schwarz inequality, we have

$$
\left\|d_{k}\right\| \geq(1-1 / \mu)\left\|F_{k}\right\| \geq(1-1 / \mu) \eta>0, \quad \forall k \geq 0,
$$


and (3.2) implies that

$$
\lim _{k \rightarrow \infty} \alpha_{k}=0
$$

On the other hand, Lemma 4 implies that $\alpha_{k} \geq \min \left\{\kappa, \frac{\rho \mu(\mu-1)}{(L+\sigma)(\mu+2)^{2}}\right\}$, which contradicts (3.4).

\section{Numerical results}

In this section, we do some numerical experiments to test the performance of Algorithm 1, herein denoted as TCGM, and compare it with the derivative-free projection method ITDM [1] and M3TFR2 method [14]. All the algorithms are coded in MATLAB R2016a. In our experiments, the algorithms are stopped whenever the inequality $\left\|F_{k}\right\| \leq 10^{-5}$ is satisfied, or the total number of iterations exceeds 5000. The parameters used in ITDM and M3TFR2 methods are set as in respective papers. The parameters in TCGM are selected as $\sigma=10^{-4}, \rho=0.5, r=10^{-3}, \mu=1.3$ and $\kappa=1$. All the algorithms are tested using the following test problems with different initial starting points and various dimensions.

Problem 1. [4]

$$
F_{i}(x)=2 c\left(x_{i}-1\right)+4 x_{i} \sum_{i=1}^{n} x_{i}^{2}-x_{i}, \quad \text { for } \quad i=1,2,3, \ldots, n, \text { and } c=10^{-5} .
$$

Problem 2. [11]

$$
F(x)=A x+g(x),
$$

where $g(x)=\left(e^{x_{1}}-1, e^{x_{2}}-1, \ldots, e^{x_{n}}-1\right)^{T}$ and

$$
A=\left(\begin{array}{ccccc}
2 & -1 & & & \\
-1 & 2 & -1 & & \\
& \ddots & \ddots & \ddots & \\
& & \ddots & \ddots & -1 \\
& & & -1 & 2
\end{array}\right)
$$

Problem 3. [1]

$$
\begin{aligned}
& F_{1}(x)=x_{1}-e^{\cos \left(\frac{x_{1}+x_{2}}{n+1}\right)}, \\
& F_{i}(x)=x_{i}-e^{\cos \left(\frac{x_{i-1}+x_{i}+x_{i+1}}{n+1}\right)}, \quad \text { for } \quad i=2,3, \ldots, n-1, \\
& F_{n}(x)=2 x_{n}-e^{\cos \left(\frac{x_{n-1}+x_{n}}{n+1}\right)} .
\end{aligned}
$$

Problem 4. [19]

$$
F_{i}(x)=e^{x_{i}}-2, \quad \text { for } \quad i=1,2,3, \ldots, n .
$$


Problem 5. [12]

$$
\begin{aligned}
& F_{1}(x)=2 x_{1}-x_{2}+e^{x_{1}}-1 \\
& F_{i}(x)=-x_{i-1}+2 x_{i}-x_{i+1}+e^{x_{i}}-1, \quad \text { for } \quad i=1,2,3, \ldots, n-1, \\
& F_{n}(x)=-x_{n-1}+2 x_{n}+e^{x_{n}}-1
\end{aligned}
$$

Problem 6. [21]

$$
\begin{aligned}
F_{2 i-1}(x) & =x_{2 i-1}+\left(\left(5-x_{2 i}\right) x_{2 i}-2\right) x_{2 i}-13 \\
F_{2 i}(x) & =x_{2 i-1}+\left(\left(1+x_{2 i}\right) x_{2 i}-14\right) x_{2 i}-29,
\end{aligned}
$$

for $i=1,2,3, \ldots, \frac{n}{2}$ ( $n$ even).

Problem \%. [21]

$$
\begin{aligned}
& F_{1}(x)=2 x_{1}+0.5 h^{2}\left(x_{1}+h\right)^{3}-x_{2}, \\
& F_{i}(x)=2 x_{i}+0.5 h^{2}\left(x_{i}+i h\right)^{3}-x_{i-1}+x_{i+1}, \text { for } \quad i=2,3, \ldots, n-1, \\
& F_{n}(x)=2 x_{n}+0.5 h^{2}\left(x_{n}+n h\right)^{3}-x_{n-1},
\end{aligned}
$$

where $h=1 /(n+1)$.

Problem 8. [2]

$$
F_{i}(x)=2 x_{i}-\sin \left(\left|x_{i}\right|\right), \quad \text { for } \quad i=1,2,3, \ldots, n .
$$

Problem 9. [21]

$$
\begin{aligned}
F_{1}(x)= & 3 x_{1}^{3}+2 x_{2}-5+\sin \left(x_{1}-x_{2}\right) \sin \left(x_{1}+x_{2}\right) \\
F_{i}(x)= & -x_{i-1} e^{x_{i-1}-x_{i}}+x_{i}\left(4+3 x_{i}^{2}\right)+2 x_{i+1} \\
& +\sin \left(x_{i}-x_{i+1}\right) \sin \left(x_{i}+x_{i+1}\right)-8, \text { for } \quad i=2,3, \ldots, n-1, \\
F_{n}(x)= & -x_{n-1} e^{x_{n-1}-x_{n}}+4 x_{n}-3 .
\end{aligned}
$$

Problem 10. [2]

$$
\begin{aligned}
& F_{1}(x)=2 x_{1}-\sin \left(x_{1}\right)-1, \\
& F_{i}(x)=-2 x_{i-1}+2 x_{i}+\sin \left(x_{i}\right)-1, \quad \text { for } \quad i=1,2,3, \ldots, n-1, \\
& F_{n}(x)=2 x_{n}+\sin \left(x_{n}\right)-1
\end{aligned}
$$

The results are presented in Tables $1-10$, where $x_{0}^{1}=(1,1, \ldots, 1)^{T}, x_{0}^{2}=$ $(-1,-1, \ldots,-1)^{T}, x_{0}^{3}=(0.1,0.1, \ldots, 0.1)^{T}$ and $x_{0}^{4}=(-0.1,-0.1, \ldots,-0.1)^{T}$. In each table, we report the dimension of the problem (DIM), the number of iterations (NI), the number of function evaluations (FE) and the CPU time in seconds. We note here that all algorithms managed to solve all the ten test functions successfully. We see that the proposed method performs better than the other methods in almost all the problems.

To have comprehensive comparisons for these methods with respect to number of iterations, number of function evaluations and the CPU time, we apply the performance profiles tool of Dolan and Moré [6] to obtain Figures 1-2. The 
Table 1. Numerical results of Problem 1.

\begin{tabular}{|c|c|c|c|c|c|c|c|c|c|c|}
\hline & & & NI & & & $\mathrm{FE}$ & & & $\mathrm{CPU}$ & \\
\hline$x_{0}$ & DIM & TCGM & M3TFR2 & ITDM & TCGM & M3TFR2 & ITDM & TCGM & M3TFR2 & ITDM \\
\hline \multirow[t]{4}{*}{$x_{0}^{1}$} & 3000 & 9 & 13 & 13 & 29 & 57 & 33 & 0.0028 & 0.0066 & 0.0045 \\
\hline & 5000 & 9 & 18 & 13 & 29 & 85 & 33 & 0.0041 & 0.0122 & 0.0068 \\
\hline & 10000 & 9 & 22 & 10 & 29 & 125 & 30 & 0.0085 & 0.0340 & 0.0122 \\
\hline & 20000 & 9 & 28 & 10 & 29 & 191 & 30 & 0.0218 & 0.1214 & 0.0216 \\
\hline \multirow[t]{4}{*}{$x_{0}^{2}$} & 3000 & 9 & 13 & 13 & 29 & 57 & 33 & 0.0029 & 0.0056 & 0.0045 \\
\hline & 5000 & 9 & 16 & 13 & 29 & 78 & 33 & 0.0040 & 0.0110 & 0.0069 \\
\hline & 10000 & 9 & 21 & 10 & 29 & 122 & 30 & 0.0108 & 0.0425 & 0.0147 \\
\hline & 20000 & 9 & 28 & 10 & 29 & 191 & 30 & 0.0144 & 0.1033 & 0.0249 \\
\hline \multirow[t]{4}{*}{$x_{0}^{3}$} & 3000 & 8 & 16 & 13 & 20 & 80 & 34 & 0.0019 & 0.0075 & 0.0044 \\
\hline & 5000 & 8 & 19 & 13 & 20 & 106 & 34 & 0.0029 & 0.0148 & 0.0070 \\
\hline & 10000 & 8 & 22 & 14 & 20 & 149 & 37 & 0.0085 & 0.0446 & 0.0196 \\
\hline & 20000 & 8 & 29 & 14 & 20 & 223 & 37 & 0.0157 & 0.1245 & 0.0351 \\
\hline \multirow[t]{4}{*}{$x_{0}^{4}$} & 3000 & 8 & 17 & 13 & 20 & 83 & 34 & 0.0030 & 0.0118 & 0.0048 \\
\hline & 5000 & 8 & 17 & 13 & 20 & 99 & 34 & 0.0029 & 0.0138 & 0.0068 \\
\hline & 10000 & 8 & 23 & 14 & 20 & 152 & 37 & 0.0054 & 0.0395 & 0.0138 \\
\hline & 20000 & 8 & 27 & 14 & 20 & 217 & 37 & 0.0104 & 0.1176 & 0.0266 \\
\hline
\end{tabular}

Table 2. Numerical results of Problem 2.

\begin{tabular}{lllllllllll}
\hline & \multicolumn{3}{c}{ NI } & & & FE & & & CPU \\
\hline$x_{0}$ & DIM & TCGM & M3TFR2 & ITDM & TCGM & M3TFR2 & ITDM & TCGM & M3TFR2 & ITDM \\
\hline$x_{0}^{1}$ & 300 & 17 & 17 & 22 & 45 & 58 & 72 & 0.0085 & 0.0298 & 0.0140 \\
& 500 & 17 & 19 & 23 & 44 & 71 & 75 & 0.0296 & 0.0772 & 0.0537 \\
& 1000 & 17 & 21 & 23 & 45 & 93 & 112 & 0.0913 & 0.1997 & 0.2398 \\
& 2000 & 16 & 24 & 23 & 40 & 125 & 75 & 0.3556 & 1.1285 & 0.6854 \\
$x_{0}^{2}$ & 300 & 17 & 21 & 23 & 40 & 80 & 72 & 0.0069 & 0.0146 & 0.0127 \\
& 500 & 18 & 21 & 26 & 44 & 89 & 121 & 0.0276 & 0.0561 & 0.0772 \\
& 1000 & 21 & 22 & 25 & 58 & 109 & 80 & 0.1163 & 0.2198 & 0.1708 \\
& 2000 & 19 & 25 & 24 & 48 & 155 & 74 & 0.4311 & 1.4178 & 0.6819 \\
$x_{0}^{3}$ & 300 & 14 & 16 & 18 & 36 & 46 & 55 & 0.0061 & 0.0087 & 0.0097 \\
& 500 & 15 & 17 & 20 & 39 & 48 & 64 & 0.0242 & 0.0299 & 0.0411 \\
& 1000 & 14 & 17 & 20 & 36 & 50 & 64 & 0.0707 & 0.1000 & 0.1301 \\
& 2000 & 14 & 17 & 19 & 35 & 48 & 57 & 0.3142 & 0.4364 & 0.5250 \\
$x_{0}^{4}$ & 300 & 14 & 16 & 18 & 37 & 47 & 58 & 0.0071 & 0.0094 & 0.0113 \\
& 500 & 14 & 16 & 19 & 36 & 47 & 61 & 0.0249 & 0.0333 & 0.0437 \\
& 1000 & 16 & 17 & 19 & 42 & 48 & 61 & 0.0831 & 0.0970 & 0.1285 \\
& 2000 & 15 & 17 & 19 & 38 & 50 & 61 & 0.3447 & 0.4539 & 0.5635 \\
\hline
\end{tabular}

Table 3. Numerical results of Problem 3.

\begin{tabular}{|c|c|c|c|c|c|c|c|c|c|c|}
\hline & & & NI & & & $\mathrm{FE}$ & & & $\mathrm{CPU}$ & \\
\hline$x_{0}$ & DIM & TCGM & M3TFR2 & ITDM & TCGM & M3TFR2 & ITDM & TCGM & M3TFR2 & ITDM \\
\hline \multirow[t]{4}{*}{$x_{0}^{1}$} & 300 & 8 & 15 & 15 & 16 & 81 & 29 & 0.0006 & 0.0093 & 0.0014 \\
\hline & 500 & 8 & 18 & 16 & 16 & 108 & 31 & 0.0009 & 0.0055 & 0.0020 \\
\hline & 1000 & 8 & 22 & 16 & 16 & 158 & 31 & 0.0014 & 0.0138 & 0.0034 \\
\hline & 2000 & 8 & 29 & 16 & 16 & 242 & 31 & 0.0026 & 0.0386 & 0.0061 \\
\hline \multirow[t]{4}{*}{$x_{0}^{2}$} & 300 & 8 & 29 & 16 & 16 & 207 & 31 & 0.0006 & 0.0075 & 0.0014 \\
\hline & 500 & 8 & 36 & 16 & 16 & 285 & 31 & 0.0009 & 0.0145 & 0.0020 \\
\hline & 1000 & 9 & 45 & 17 & 18 & 420 & 33 & 0.0016 & 0.0365 & 0.0036 \\
\hline & 2000 & 9 & 61 & 17 & 18 & 639 & 33 & 0.0029 & 0.1014 & 0.0065 \\
\hline \multirow[t]{4}{*}{$x_{0}^{3}$} & 300 & 8 & 20 & 16 & 16 & 131 & 31 & 0.0006 & 0.0047 & 0.0014 \\
\hline & 500 & 8 & 28 & 16 & 16 & 188 & 31 & 0.0009 & 0.0096 & 0.0020 \\
\hline & 1000 & 8 & 33 & 16 & 16 & 276 & 31 & 0.0014 & 0.0243 & 0.0034 \\
\hline & 2000 & 9 & 46 & 17 & 18 & 423 & 33 & 0.0029 & 0.0673 & 0.0065 \\
\hline \multirow[t]{4}{*}{$x_{0}^{4}$} & 300 & 8 & 27 & 16 & 16 & 164 & 31 & 0.0006 & 0.0060 & 0.0014 \\
\hline & 500 & 8 & 26 & 16 & 16 & 198 & 31 & 0.0009 & 0.0101 & 0.0020 \\
\hline & 1000 & 9 & 35 & 16 & 18 & 300 & 31 & 0.0016 & 0.0261 & 0.0034 \\
\hline & 2000 & 9 & 49 & 17 & 18 & 463 & 33 & 0.0029 & 0.0737 & 0.0065 \\
\hline
\end{tabular}


Table 4. Numerical results of Problem 4.

\begin{tabular}{lllllllllll}
\hline & \multicolumn{3}{c}{ NI } & \multicolumn{3}{c}{ FE } & \multicolumn{3}{c}{ CPU } \\
\hline$x_{0}$ & DIM & TCGM & M3TFR2 & ITDM & TCGM & M3TFR2 & ITDM & TCGM & M3TFR2 & ITDM \\
\hline$x_{0}^{1}$ & 300 & 7 & 5 & 10 & 20 & 11 & 28 & 0.0005 & 0.0030 & 0.0008 \\
& 500 & 7 & 5 & 10 & 20 & 13 & 28 & 0.0005 & 0.0004 & 0.0010 \\
& 1000 & 7 & 6 & 11 & 20 & 18 & 31 & 0.0007 & 0.0007 & 0.0015 \\
& 2000 & 8 & 8 & 11 & 23 & 28 & 31 & 0.0012 & 0.0014 & 0.0022 \\
& & & & & & & & & & \\
$x_{0}^{2}$ & 300 & 7 & 19 & 10 & 18 & 104 & 26 & 0.0004 & 0.0024 & 0.0008 \\
& 500 & 7 & 21 & 11 & 18 & 135 & 29 & 0.0005 & 0.0034 & 0.0011 \\
& 1000 & 8 & 26 & 11 & 21 & 193 & 29 & 0.0008 & 0.0062 & 0.0014 \\
& 2000 & 8 & 35 & 11 & 21 & 295 & 29 & 0.0010 & 0.0130 & 0.0021 \\
& & & & & & & & & & \\
$x_{0}^{3}$ & 300 & 8 & 13 & 10 & 22 & 46 & 27 & 0.0005 & 0.0011 & 0.0008 \\
& 500 & 8 & 12 & 11 & 22 & 43 & 30 & 0.0006 & 0.0012 & 0.0011 \\
& 1000 & 8 & 14 & 11 & 22 & 60 & 30 & 0.0008 & 0.0020 & 0.0014 \\
& 2000 & 8 & 18 & 11 & 22 & 87 & 30 & 0.0011 & 0.0040 & 0.0021 \\
& & & & & & & & & \\
$x_{0}^{4}$ & 300 & 8 & 13 & 11 & 22 & 51 & 30 & 0.0005 & 0.0012 & 0.0009 \\
& 500 & 8 & 15 & 11 & 22 & 62 & 30 & 0.0006 & 0.0016 & 0.0010 \\
& 1000 & 8 & 16 & 12 & 22 & 78 & 33 & 0.0008 & 0.0026 & 0.0015 \\
& 2000 & 9 & 21 & 12 & 25 & 118 & 33 & 0.0012 & 0.0053 & 0.0023 \\
\hline
\end{tabular}

Table 5. Numerical results of Problem 5.

\begin{tabular}{lllllllllll}
\hline & \multicolumn{3}{c}{ NI } & & & \multicolumn{2}{l}{ FE } & & \multicolumn{2}{c}{ CPU } \\
\hline$x_{0}$ & DIM & TCGM & M3TFR2 & ITDM & TCGM & M3TFR2 & ITDM & TCGM & M3TFR2 & ITDM \\
\hline$x_{0}^{1}$ & 3000 & 17 & 27 & 22 & 45 & 159 & 69 & 0.0097 & 0.0326 & 0.0165 \\
& 5000 & 19 & 33 & 23 & 50 & 222 & 72 & 0.0142 & 0.0724 & 0.0258 \\
& 10000 & 18 & 41 & 21 & 46 & 331 & 64 & 0.0205 & 0.1862 & 0.0343 \\
& 20000 & 17 & 55 & 25 & 42 & 507 & 80 & 0.0416 & 0.5543 & 0.0997 \\
$x_{0}^{2}$ & 3000 & 19 & 30 & 26 & 48 & 200 & 81 & 0.0104 & 0.0447 & 0.0198 \\
& 5000 & 19 & 32 & 25 & 47 & 252 & 78 & 0.0160 & 0.0680 & 0.0301 \\
& 10000 & 19 & 44 & 28 & 47 & 389 & 129 & 0.0279 & 0.2348 & 0.0753 \\
& 20000 & 17 & 57 & 26 & 40 & 577 & 83 & 0.0705 & 0.6020 & 0.1022 \\
$x_{0}^{3}$ & 3000 & 14 & 17 & 20 & 35 & 51 & 61 & 0.0051 & 0.0080 & 0.0119 \\
& 5000 & 14 & 16 & 20 & 35 & 46 & 61 & 0.0080 & 0.0144 & 0.0213 \\
& 10000 & 14 & 17 & 19 & 34 & 52 & 94 & 0.0153 & 0.0238 & 0.0509 \\
& 20000 & 14 & 13 & 20 & 34 & 44 & 59 & 0.0287 & 0.0423 & 0.0603 \\
$x_{0}^{4}$ & 3000 & 15 & 18 & 15 & 38 & 53 & 44 & 0.0056 & 0.0078 & 0.0076 \\
& 5000 & 15 & 17 & 21 & 38 & 50 & 70 & 0.0087 & 0.0115 & 0.0188 \\
& 10000 & 16 & 18 & 19 & 41 & 58 & 59 & 0.0225 & 0.0259 & 0.0405 \\
& 20000 & 16 & 17 & 20 & 42 & 58 & 63 & 0.0470 & 0.0496 & 0.0793 \\
\hline
\end{tabular}

Table 6. Numerical results of Problem 6 .

\begin{tabular}{lllllllllll}
\hline & \multicolumn{3}{c}{ NI } & & \multicolumn{3}{c}{ FE } & & CPU \\
\hline$x_{0}$ & DIM & TCGM & M3TFR2 & ITDM & TCGM & M3TFR2 & ITDM & TCGM & M3TFR2 & ITDM \\
\hline$x_{0}^{1}$ & 300 & 181 & 204 & 624 & 984 & 878 & 9849 & 0.0193 & 0.0220 & 0.1957 \\
& 500 & 125 & 210 & 1083 & 678 & 945 & 17573 & 0.0166 & 0.0253 & 0.4370 \\
& 1000 & 155 & 128 & 384 & 835 & 806 & 5937 & 0.0301 & 0.0305 & 0.2251 \\
& 2000 & 231 & 196 & 881 & 1296 & 1285 & 14023 & 0.0770 & 0.0812 & 0.8993 \\
$x_{0}^{2}$ & 300 & 185 & 197 & 794 & 1030 & 895 & 12665 & 0.0205 & 0.0189 & 0.2527 \\
& 500 & 97 & 194 & 930 & 515 & 1014 & 14747 & 0.0126 & 0.0263 & 0.3688 \\
& 1000 & 189 & 218 & 729 & 1046 & 1365 & 11549 & 0.0374 & 0.0516 & 0.4370 \\
& 2000 & 124 & 270 & 600 & 675 & 1874 & 9327 & 0.0400 & 0.1167 & 0.5942 \\
$x_{0}^{3}$ & 300 & 198 & 252 & 524 & 1093 & 1075 & 8202 & 0.0217 & 0.0228 & 0.1625 \\
& 500 & 144 & 158 & 832 & 772 & 852 & 13048 & 0.0189 & 0.0221 & 0.3267 \\
& 1000 & 154 & 242 & 845 & 842 & 1314 & 13223 & 0.0302 & 0.0504 & 0.5015 \\
& 2000 & 193 & 229 & 680 & 1068 & 1543 & 10564 & 0.0628 & 0.0958 & 0.6666 \\
$x_{0}^{4}$ & 300 & 117 & 157 & 824 & 622 & 775 & 13077 & 0.0123 & 0.0162 & 0.2595 \\
& 500 & 114 & 303 & 825 & 604 & 1350 & 13067 & 0.0147 & 0.0355 & 0.3267 \\
& 1000 & 86 & 235 & 815 & 454 & 1283 & 12914 & 0.0163 & 0.0495 & 0.4895 \\
& 2000 & 109 & 231 & 868 & 578 & 1606 & 13877 & 0.0343 & 0.0998 & 0.8735 \\
\hline
\end{tabular}


Table 7. Numerical results of Problem 7.

\begin{tabular}{lllllllllll}
\hline & \multicolumn{3}{c}{ NI } & \multicolumn{3}{c}{ FE } & & \multicolumn{3}{c}{ CPU } \\
\hline$x_{0}$ & DIM & TCGM & M3TFR2 & ITDM & TCGM & M3TFR2 & ITDM & TCGM & M3TFR2 & ITDM \\
\hline$x_{0}^{1}$ & 300 & 60 & 119 & 37 & 178 & 370 & 140 & 0.0117 & 0.0246 & 0.0096 \\
& 500 & 60 & 112 & 37 & 178 & 357 & 139 & 0.0178 & 0.0360 & 0.0145 \\
& 1000 & 60 & 108 & 36 & 178 & 364 & 134 & 0.0330 & 0.0680 & 0.0259 \\
& 2000 & 60 & 100 & 33 & 178 & 369 & 122 & 0.0632 & 0.1323 & 0.0459 \\
& & & & & & & & & & \\
$x_{0}^{2}$ & 300 & 60 & 119 & 37 & 178 & 370 & 140 & 0.0119 & 0.0250 & 0.0097 \\
& 500 & 60 & 112 & 37 & 178 & 357 & 139 & 0.0178 & 0.0361 & 0.0145 \\
& 1000 & 60 & 108 & 36 & 178 & 364 & 134 & 0.0329 & 0.0679 & 0.0258 \\
& 2000 & 60 & 100 & 33 & 178 & 369 & 122 & 0.0631 & 0.1319 & 0.0453 \\
$x_{0}^{3}$ & 300 & 43 & 76 & 29 & 127 & 227 & 106 & 0.0085 & 0.0152 & 0.0074 \\
& 500 & 43 & 74 & 28 & 127 & 221 & 105 & 0.0127 & 0.0223 & 0.0109 \\
& 1000 & 43 & 72 & 29 & 127 & 215 & 106 & 0.0234 & 0.0401 & 0.0205 \\
& 2000 & 43 & 70 & 28 & 127 & 209 & 103 & 0.0450 & 0.0748 & 0.0381 \\
$x_{0}^{4}$ & 300 & 43 & 76 & 29 & 127 & 227 & 106 & 0.0084 & 0.0151 & 0.0073 \\
& 500 & 43 & 74 & 28 & 127 & 221 & 105 & 0.0128 & 0.0224 & 0.0110 \\
& 1000 & 43 & 72 & 29 & 127 & 215 & 106 & 0.0234 & 0.0402 & 0.0204 \\
& 2000 & 43 & 70 & 28 & 127 & 209 & 103 & 0.0450 & 0.0748 & 0.0382 \\
\hline
\end{tabular}

Table 8. Numerical results of Problem 8.

\begin{tabular}{|c|c|c|c|c|c|c|c|c|c|c|}
\hline & & & NI & & & $\mathrm{FE}$ & & & $\mathrm{CPU}$ & \\
\hline$x_{0}$ & DIM & TCGM & M3TFR2 & ITDM & TCGM & M3TFR2 & ITDM & TCGM & M3TFR2 & ITDM \\
\hline \multirow[t]{4}{*}{$x_{0}^{1}$} & 3000 & 8 & 23 & 16 & 16 & 160 & 31 & 0.0011 & 0.0092 & 0.0034 \\
\hline & 5000 & 8 & 27 & 16 & 16 & 209 & 31 & 0.0015 & 0.0164 & 0.0050 \\
\hline & 10000 & 8 & 39 & 16 & 16 & 337 & 31 & 0.0028 & 0.0434 & 0.0092 \\
\hline & 20000 & 9 & 52 & 17 & 18 & 511 & 33 & 0.0053 & 0.1367 & 0.0179 \\
\hline \multirow[t]{4}{*}{$x_{0}^{2}$} & 3000 & 15 & 26 & 15 & 57 & 180 & 57 & 0.0040 & 0.0129 & 0.0056 \\
\hline & 5000 & 15 & 31 & 16 & 57 & 234 & 61 & 0.0054 & 0.0229 & 0.0084 \\
\hline & 10000 & 15 & 43 & 16 & 57 & 362 & 61 & 0.0080 & 0.0532 & 0.0129 \\
\hline & 20000 & 15 & 58 & 16 & 57 & 553 & 61 & 0.0136 & 0.1349 & 0.0255 \\
\hline \multirow[t]{4}{*}{$x_{0}^{3}$} & 3000 & 7 & 4 & 13 & 14 & 10 & 25 & 0.0012 & 0.0008 & 0.0034 \\
\hline & 5000 & 7 & 5 & 14 & 14 & 14 & 27 & 0.0015 & 0.0014 & 0.0048 \\
\hline & 10000 & 8 & 7 & 14 & 16 & 24 & 27 & 0.0028 & 0.0037 & 0.0083 \\
\hline & 20000 & 8 & 7 & 14 & 16 & 28 & 27 & 0.0048 & 0.0071 & 0.0147 \\
\hline \multirow[t]{4}{*}{$x_{0}^{4}$} & 3000 & 12 & 10 & 13 & 45 & 29 & 49 & 0.0030 & 0.0022 & 0.0046 \\
\hline & 5000 & 12 & 9 & 13 & 45 & 27 & 49 & 0.0037 & 0.0025 & 0.0062 \\
\hline & 10000 & 13 & 10 & 14 & 49 & 34 & 53 & 0.0060 & 0.0047 & 0.0106 \\
\hline & 20000 & 13 & 12 & 14 & 49 & 44 & 53 & 0.0113 & 0.0109 & 0.0203 \\
\hline
\end{tabular}

Table 9. Numerical results of Problem 9.

\begin{tabular}{lllllllllll}
\hline & \multicolumn{3}{c}{ NI } & & \multicolumn{3}{l}{ FE } & & & CPU \\
\hline$x_{0}$ & DIM & TCGM & M3TFR2 & ITDM & TCGM & M3TFR2 & ITDM & TCGM & M3TFR2 & ITDM \\
\hline$x_{0}^{1}$ & 3000 & 1 & 1 & 1 & 1 & 1 & 1 & 0.0002 & 0.0002 & 0.0002 \\
& 5000 & 1 & 1 & 1 & 1 & 1 & 1 & 0.0003 & 0.0004 & 0.0003 \\
& 10000 & 1 & 1 & 1 & 1 & 1 & 1 & 0.0007 & 0.0007 & 0.0007 \\
& 20000 & 1 & 1 & 1 & 1 & 1 & 1 & 0.0013 & 0.0013 & 0.0013 \\
$x_{0}^{2}$ & 3000 & 19 & 53 & 19 & 93 & 447 & 111 & 0.0221 & 0.1023 & 0.0289 \\
& 5000 & 19 & 65 & 23 & 93 & 586 & 136 & 0.0529 & 0.2467 & 0.0640 \\
& 10000 & 19 & 81 & 23 & 93 & 839 & 138 & 0.0852 & 0.6982 & 0.1223 \\
& 20000 & 19 & 109 & 24 & 93 & 1266 & 147 & 0.1505 & 2.1338 & 0.3055 \\
$x_{0}^{3}$ & 3000 & 21 & 31 & 19 & 106 & 187 & 110 & 0.0431 & 0.0489 & 0.0282 \\
& 5000 & 21 & 35 & 19 & 106 & 243 & 110 & 0.0475 & 0.1227 & 0.0466 \\
& 10000 & 21 & 46 & 21 & 105 & 368 & 124 & 0.1213 & 0.3983 & 0.1271 \\
& 20000 & 21 & 60 & 19 & 107 & 544 & 111 & 0.2026 & 0.9904 & 0.2261 \\
$x_{0}^{4}$ & 3000 & 19 & 34 & 22 & 91 & 230 & 131 & 0.0412 & 0.0693 & 0.0356 \\
& 5000 & 21 & 39 & 20 & 103 & 299 & 117 & 0.0614 & 0.1698 & 0.0552 \\
& 10000 & 20 & 50 & 20 & 98 & 439 & 119 & 0.0856 & 0.3457 & 0.1516 \\
& 20000 & 20 & 68 & 21 & 98 & 667 & 124 & 0.1671 & 1.2313 & 0.2964 \\
\hline
\end{tabular}


Table 10. Numerical results of Problem 10.

\begin{tabular}{lllllllllll}
\hline & \multicolumn{3}{c}{ NI } & & \multicolumn{3}{c}{ FE } & & \multicolumn{2}{c}{ CPU } \\
\hline$x_{0}$ & DIM & TCGM & M3TFR2 & ITDM & TCGM & M3TFR2 & ITDM & TCGM & M3TFR2 & ITDM \\
\hline$x_{0}^{1}$ & 3000 & 47 & 55 & 46 & 156 & 231 & 240 & 0.0182 & 0.0293 & 0.0438 \\
& 5000 & 47 & 60 & 49 & 156 & 282 & 270 & 0.0390 & 0.0554 & 0.0783 \\
& 10000 & 47 & 68 & 47 & 158 & 371 & 203 & 0.0638 & 0.1485 & 0.1183 \\
& 20000 & 47 & 80 & 53 & 158 & 515 & 287 & 0.1047 & 0.4317 & 0.2471 \\
$x_{0}^{2}$ & 3000 & 50 & 66 & 56 & 163 & 324 & 266 & 0.0226 & 0.0383 & 0.0470 \\
& 5000 & 50 & 75 & 56 & 165 & 412 & 312 & 0.0382 & 0.0929 & 0.1007 \\
& 10000 & 50 & 86 & 53 & 165 & 558 & 228 & 0.0632 & 0.2602 & 0.1246 \\
& 20000 & 51 & 105 & 54 & 170 & 799 & 282 & 0.1357 & 0.6699 & 0.3113 \\
$x_{0}^{3}$ & 3000 & 44 & 43 & 54 & 142 & 129 & 278 & 0.0179 & 0.0255 & 0.0422 \\
& 5000 & 44 & 43 & 52 & 142 & 130 & 255 & 0.0252 & 0.0241 & 0.0527 \\
& 10000 & 44 & 44 & 45 & 142 & 137 & 274 & 0.0804 & 0.0732 & 0.1359 \\
& 20000 & 44 & 47 & 50 & 144 & 149 & 244 & 0.0962 & 0.1259 & 0.2088 \\
$x_{0}^{4}$ & 3000 & 46 & 46 & 47 & 150 & 150 & 210 & 0.0237 & 0.0181 & 0.0287 \\
& 5000 & 46 & 49 & 47 & 150 & 168 & 220 & 0.0347 & 0.0328 & 0.0489 \\
& 10000 & 47 & 52 & 50 & 155 & 192 & 315 & 0.0682 & 0.0813 & 0.1844 \\
& 20000 & 46 & 56 & 50 & 152 & 233 & 228 & 0.1068 & 0.1990 & 0.2056 \\
\hline
\end{tabular}

Dolan and Moré [6] performance profiles procedure is a tool for evaluating and comparing the performances of iterative methods. The profile of each method is measured according to the ratio of its computational outcome compared to the computational outcome of the best presented method. Figures 1-2 clearly show that TCGM method is the most efficient as compared to the other two methods.

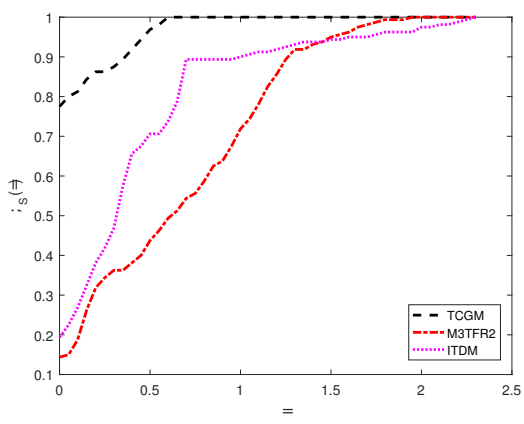

Figure 1. Iterations performance profile

\section{Conclusions}

In this paper, we proposed a three-term conjugate gradient-based method $(T C G M)$ for solving systems of large-scale nonlinear monotone equations. The proposed method is free from derivative evaluations and also satisfies the sufficient descent condition independent of any line search. Its global convergence was also established. The proposed algorithm was tested on some benchmark problems with different starting points and different dimensions and the numerical results show that the method is very efficient. 


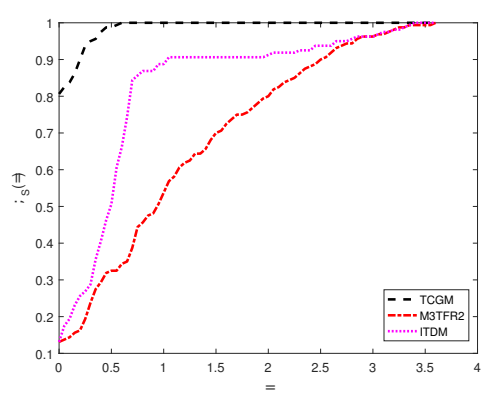

a)

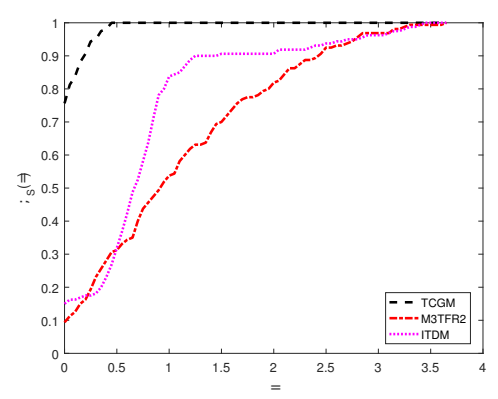

b)

Figure 2. Results of numerical experiments: a) function evaluations performance profile b) CPU time performance profile

\section{References}

[1] A.B. Abubakar and P. Kumam. An improved three-term derivative-free method for solving nonlinear equations. Comp. Appl. Math., 37(5):6760-6773, 2018. https://doi.org/10.1007/s40314-018-0712-5.

[2] A.B. Abubakar and P. Kumam. A descent Dai-Liao conjugate gradient method for nonlinear equations. Numer. Algor., 81(1):197-210, 2019. https://doi.org/10.1007/s11075-018-0541-z.

[3] B. Baluch, Z. Salleh, A. Alhawarat and U.A.M. Roslan. A new modified three-term conjugate gradient method with sufficient descent property and its global convergence. J. Math., 2017(Article ID 2715854):12 pages, 2017. https://doi.org/10.1155/2017/2715854.

[4] Y. Ding, Y. Xiao and J. Li. A class of conjugate gradient methods for convex constrained monotone equations. Optim., 66(12):2309-2328, 2017. https://doi.org/10.1080/02331934.2017.1372438.

[5] S.P. Dirkse and M.C. Ferris. MCPLIB: A collection of nonlinear mixed complimentary problems. Optim. Methods Softw., 5:319-345, 1995. https://doi.org/10.1080/10556789508805619.

[6] E.D. Dolan and J.J. More. Benchmarking optimization software with performance profiles. Math. Program., 91(2):201-213, 2002. https://doi.org/10.1007/s101070100263.

[7] D. Feng, M. Sun and X. Wang. A family of conjugate gradient methods for large-scale nonlinear equations. J. Inequal. Appl., 2017(236):1-8, 2017. https://doi.org/10.1186/s13660-017-1510-0.

[8] Q. Hu, Y. Xiao and Q. Wang. Non-smooth equations based methods for $l_{1}$-norm problems with applications to compressed sensing. Nonlinear Anal., 74:35703577, 2011. https://doi.org/10.1016/j.na.2011.02.040.

[9] M.A.H. Ibrahim, M. Mamat and W.J. Leong. The hybrid BFGS-CG method in solving unconstrained optimization problems. Abs. Appl. Anal., 2014(Article ID 507102):6 pages, 2014. https://doi.org/10.1155/2014/507102.

[10] J. Liu and Y. Feng. A derivative-free iterative method for nonlinear monotone equations with convex constraints. Numer. Algor., 82(1):245-262, 2019. https://doi.org/10.1007/s11075-018-0603-2. 
[11] J. Liu and S. Li. Spectral DY-type projection method for nonlinear monotone system of equations. J. Comput. Math., 33(4):341-355, 2015. https://doi.org/10.4208/jcm.1412-m4494.

[12] J. Liu and S. Li. A three-term derivative-free projection method for nonlinear monotone system of equations. Calcolo, 53(3):427-450, 2016. https://doi.org/10.1007/s10092-015-0156-x.

[13] K. Meintjes and A.P. Morgan. A methodology for solving chemical equilibrium systems. Appl. Math. Comput., 22:333-361, 1987. https://doi.org/10.1016/00963003(87)90076-2.

[14] Z. Papp and S. Rapajić. FR type methods for systems of large-scale nonlinear monotone equations. Appl. Math. Comput., 269:816-823, 2015. https://doi.org/10.1016/j.amc.2015.08.002.

[15] M.A.K. Shiker and K. Amini. A new projection-based algorithm for solving a large-scale nonlinear system of monotone equations. Croat. Oper. Res. Rev, 9:63-73, 2018. https://doi.org/10.17535/crorr.2018.0006.

[16] M.V. Solodov and B.F. Svaiter. A globally convergent inexact Newton method for systems of monotone equations. In M. Fukushima and L. Qi(Eds.), Reformulation: Nonsmooth, Piecewise Smooth, Semismooth and Smoothing Methods, volume 22 of Applied Optimization, pp. 355-369, Boston, MA, 1998. Springer. https://doi.org/10.1007/978-1-4757-6388-1_18.

[17] P.S. Stanimirović, B. Ivanov, S. Djordjević and I. Brajević. New hybrid conjugate gradient and Broyden-Fletcher-Goldfarb-Shanno conjugate gradient methods. $J$. Optim. Theory Appl., 178(3):860-884, 2018. https://doi.org/10.1007/s10957018-1324-3.

[18] M. Sun and J. Liu. Three derivative-free projection methods for nonlinear equations with convex constraints. J. Appl. Math. Comp., 47(1-2):265-276, 2015. https://doi.org/10.1007/s12190-014-0774-5.

[19] S. Wang and H. Guan. A scaled conjugate gradient method for solving monotone nonlinear equations with convex constraints. J. Appl. Math., 2013(Article ID 286486):7 pages, 2013. https://doi.org/10.1155/2013/286486.

[20] X.Y. Wang, S.J. Li and X.P. Kou. A self-adaptive three-term conjugate gradient method for monotone nonlinear equations with convex constraints. Calcolo, 53(2):133-145, 2016. https://doi.org/10.1007/s10092-015-0140-5.

[21] G. Yuan and M. Zhang. A three-terms Polak-Ribiere-Polyak conjugate gradient algorithm for large-scale nonlinear equations. J. Comput. Appl. Math., 286:186195, 2015. https://doi.org/10.1016/j.cam.2015.03.014.

[22] Y. Zheng and B. Zheng. Two new Dai-Liao-type conjugate gradient methods for unconstrained optimization problems. J. Optim. Theory. Appl., 175(2):502-509, 2017. https://doi.org/10.1007/s10957-017-1140-1. 